\title{
Формирование сингулярных (001) террас на поверхности монокристаллических НРНТ алмазных подложек
}

\author{
(C) П.А. Юнин ${ }^{*+\bullet}$, Ю.Н. Дроздов ${ }^{*}$, В.В. Чернов ${ }^{+}$, В.А. Исаев ${ }^{+}$, \\ C.А. Богданов ${ }^{+}$, А.Б. Мучников ${ }^{+}$
}

* Институт фризики микроструктур Российской академии наук, 603950 Нижний Новгород, Россия

+ Институт прикладной физики Российской академии наук, 603950 Нижний Новгород, Россия

- Нижегородский государственный университет им. Н.И. Лобачевского, 603950 Нижний Новгород, Россия

E-mail: yunin@ipmras.ru

(Получена 27 апреля 2016 г. Принята к печати 10 мая 2016 г.)

Обсуждаются способы экспериментальной диагностики и управления величиной отклонения плоскости поверхности монокристаллических алмазных НРНT (High Pressure High Temperature) пластин относительно кристаллографических плоскостей (001). Такие пластины (подложки) используются для гомоэпитаксиального выращивания как легированных, так и нелегированных пленок CVD алмаза. При травлении HPHT подложек в плазме СВЧ разряда в чистом водороде либо в водород-кислородной смеси наблюдается формирование сингулярных террас с ориентацией поверхности (001). Это явление может быть использовано как для экспресс-анализа ориентации подложки и повышения точности контроля угла отклонения при шлифовке подложек, так и для получения макроскопических сингулярных (001) участков монокристаллических алмазных подложек.

\section{1. Введение}

По совокупности свойств монокристаллический CVD (Chemical Vapor Deposition) алмаз является исключительным материалом, поэтому уже в настоящее время он активно используется в электронике, оптике, акустоэлектронике, физике высоких энергий и ряде других областей [1,2]. Рост алмазных пленок „электронного“ качества в основном осуществляется гомоэпитаксиально на монокристаллических алмазных НРНТ подложках. Свойства гомоэпитаксиальных пленок сильно зависят от параметров подложки. Важным параметром является величина разориентации подложки, т.е. отклонение плоскости поверхности подложки от определенной кристаллографической плоскости. Или же, переходя в описании от плоскостей к нормалям, угол разориентации - это угол между нормалью к поверхности подложки и кристаллографической осью [001] (см. рис. 1).

Свойства эпитаксиально выращенного слоя CVD алмаза могут заметно различаться даже для роста на областях одной и той же блочной подложки с различными углами разориентации [3-5]. Таким образом, важной частью процесса роста эпитаксиальных пленок алмаза является этап получения алмазных подложек с известной величиной отклонения кристаллографических плоскостей (001) от плоскости поверхности (с точностью не хуже $0.05^{\circ}$ ). Такая задача требует разработки как методик прецизионной диагностики ориентации кристаллографических осей, так и методов предварительной обработки поверхности алмазной подложки.

\section{2. Методика эксперимента}

Для гомоэпитаксиального CVD роста алмазных пленок были использованы НРНТ (High Pressure High Temperature) подложки фирмы New Diamond Technologies (г. Сестрорецк, Россия) в форме прямоугольного параллелепипеда с размерами $3.5 \times 3.5 \times 0.5$ мм. Подложки были вырезаны таким образом, чтобы поверхность была приблизительно параллельна кристаллографическим плоскостям (001). Угол разориентации используемых подложек варьируется в пределах до $6^{\circ}$ и заранее неизвестен.

Диагностика ориентации кристаллографических осей относительно геометрической формы алмазных подложек проводилась методом рентгеновской дифрактомет-

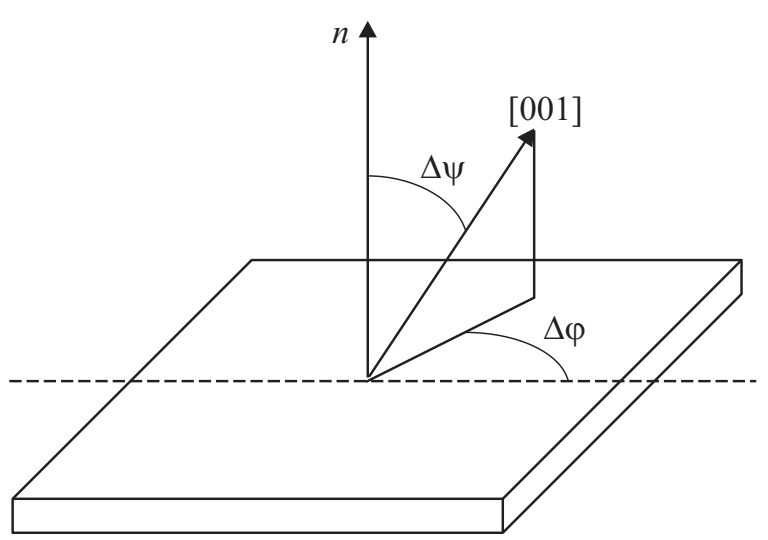

Рис. 1. Иллюстрация обозначения эйлеровых углов разориентации $(\Delta \varphi, \Delta \psi)$ между нормалью к поверхности алмазной подложки и кристаллографическим направлением [001]. 
рии (РД) на многофункциональном рентгеновском дифрактометре Bruker D8 Discover (Bruker AXS). Шлифовка и полировка поверхности монокристаллических алмазных подложек проводилась на ограночном станке, применяемом в ювелирной промышленности.

Одним из этапов подготовки поверхности алмазной подложки для гомоэпитаксиального роста является ее травление непосредственно в камере MPACVD (Microwave Plasma Assisted Chemical Vapor Deposition) реактора [6]. Травление осуществлялось в чистом водороде, либо водород-кислородной смеси [5]. Диагностика морфологии подложек после травления в плазме СВЧ разряда проводилась на интерференционном микроскопе белого света (ИМБС) Zygo New View 7300 и атомносиловом микроскопе (ACM) NT-MDT Nanoeducator II.

\section{3. Результаты и обсуждение}

Для определения ориентации кристаллографических осей алмазной подложки относительно ее геометрической формы методом РД необходимо установить соответствие как между кристаллографической системой координат и осями гониометра, так и между осями гониометра и геометрической формой образца $[7,8]$. Первая часть задачи решается нахождением положений углов гониометра, при которых наблюдается дифракция рентгеновского излучения на выбранных семействах атомных плоскостей (в данном случае для подложки с ориентацией (001) использовалось отражение (004) алмаза), и благодаря точности подвижки углов современных дифрактометров эта процедура осуществляется с очень малой погрешностью. Гониометр дифрактометра Bruker D8 Discover позволяет с точностью до тысячных долей градуса осуществлять автоматизированное вращение подложек как в своей плоскости (угол $\varphi$ ), так и наклон в перпендикулярной плоскости (угол $\psi)$. Вторая часть - установление соответствия между осями гониометра и геометрической формой образца - определяется методикой юстировки образца относительно гониометра и дает наибольший вклад в погрешность определения угла разориентации. Таким образом, точность определения угла разориентации $\Delta \psi$ будет в большей степени определяться точностью вывода нормали к поверхности кристалла параллельно $\varphi$-оси вращения гониометра.

Для прецизионного метода юстировки была адаптирована телескопическая приставка из комплекта дифрактометра ДРОН-4, позволяющая наблюдать отражение светового пятна от зеркальной поверхности образца. Свет после конденсора коллимируется крестом щелей, попадает на зеркальную поверхность образца и отражается. Отраженный световой крест наблюдается в телескопическую зрительную трубу. Двумя независимыми углами наклона держателя образца достигается неподвижность креста при вращении образца вокруг оси $\varphi$, что свидетельствует о параллельности оси $\varphi$ и нормали к зеркальной поверхности образца. Погрешность юстировки по углу разориентации $\psi$ обусловлена конечной угловой шириной отраженного креста, которая составляет $0.02^{\circ}$ для алмазных подложек с латеральными размерами $3.5 \times 3.5$ мм. Использование специально изготовленного держателя образца позволило также с точностью до $5^{\circ}$ определять направление отклонения в плоскости по углу $\varphi$ относительно боковых граней подложки.

Для контролируемого получения подложек с заданной величиной разориентации отрабатывалась методика их шлифовки и полировки на ограночном станке. Найденные методом РД углы отклонения кристаллографического направления [001] относительно нормали к поверхности $(\Delta \varphi, \Delta \psi)$ (рис. $1,2, a)$ использовались в дальнейшем при шлифовке поверхности образца для изменения величины отклонения. При закреплении образца перед шлифовкой на ограночном станке для юстировки использовалось отражение лазерного луча от зеркальной поверхности образца, что дает погрешность юстировки на уровне $0.1^{\circ}$. Процедуры шлифовки и ориентировки на дифрактометре могут быть повторены неоднократно для получения желаемой величины разориентации. Для проведения экспериментов с помощью шлифовки были получены подложки с различной заданной величиной разориентации от 0.2 до $4^{\circ}$, которые могут быть использованы для получения совершенных CVD слоев алмаза [4], в том числе и содержащих примесные центры азота.

Эффективным способом удаления нарушенного механической шлифовкой слоя подложек монокристаллического алмаза перед ростом пленок в CVD peакторе является травление в плазме СВЧ разряда в чистом водороде, либо водород-кислородной смеси [5]. При травлении подложек с плотностью дислокаций менее $10^{4} \mathrm{~cm}^{-2}$ наблюдалось интересное явление формирование массивов клинообразных мезаструктур, имеющих широкую, гладкую, слабо отклоненную от поверхности террасу и одинаковую ориентацию террас по всей поверхности подложки. Стоит отметить, что формирование таких террас наблюдалось только для подложек с низкой плотностью дислокаций. Также могут быть подобраны режимы травления, при которых не наблюдается развития этого эффекта. Возникло предположение, что форма террас определяется кристаллографическими плоскостями. Для проверки предположения было выполнено сравнение морфологии поверхности полученных образцов с массивами террас и результатов РД определения разориентации подложек.

Исследования морфологии поверхности проводились на интерференционном микроскопе белого света Zygo New View 7300 и атомно-силовом микроскопе NT-MDT Nanoeducator II. Результаты исследований для одного из образцов приведены на рис. $2, b, 3, a, b$. Видно, что на приведенной в качестве примера подложке S02 сформировались террасы с характерными размерами в 

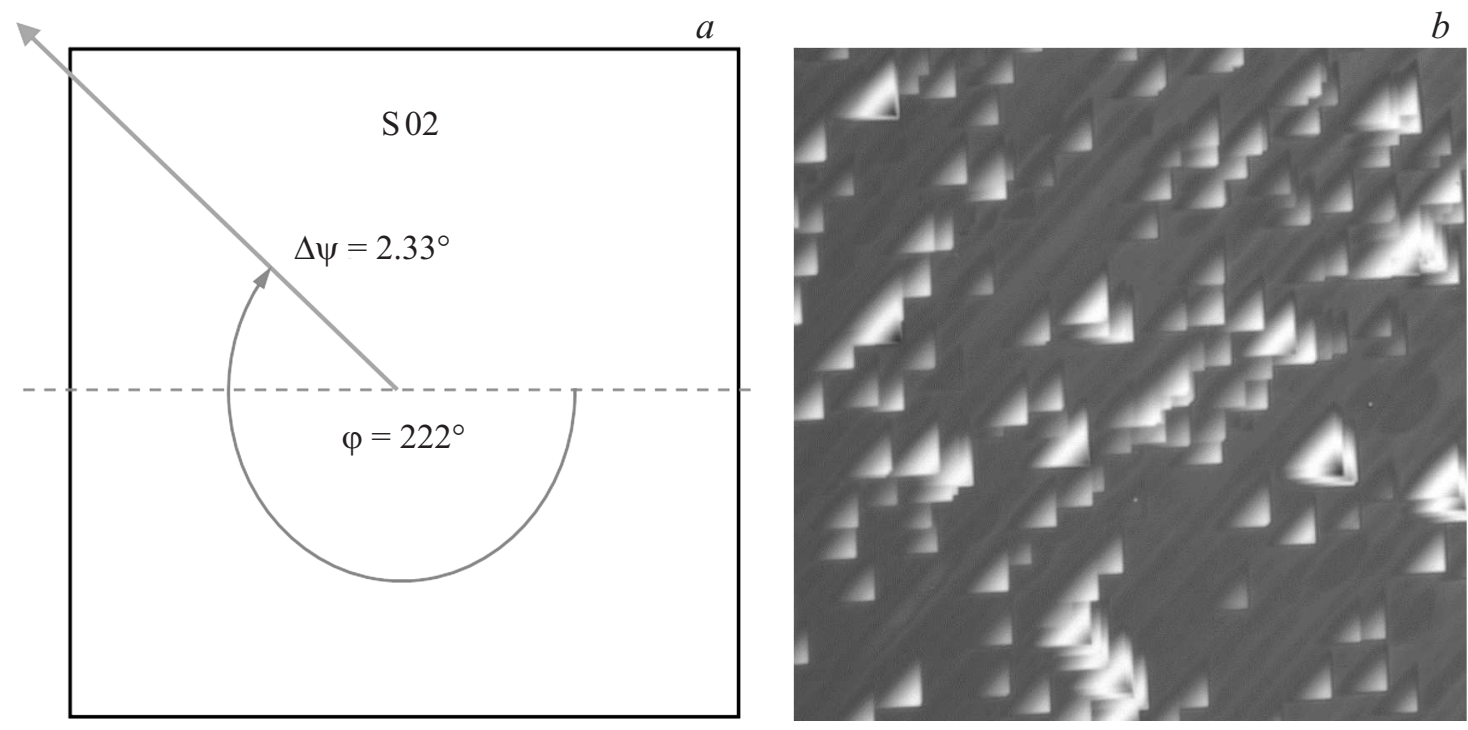

Рис. 2. $a-$ направление в плоскости $(\Delta \varphi)$ и величина отклонения от нормали к поверхности $(\Delta \psi)$ кристаллографической оси [001] подложки S02, определенные методом РД; $b$ - поверхность алмазной подложки S02 после травления в водородкислородной смеси, кадр с интерференционного микроскопа Zygo New View 7300, размер кадра $0.11 \times 0.11$ мм. Видно, что ориентация террас в плоскости соответствует РД данным на рис. 2, a.
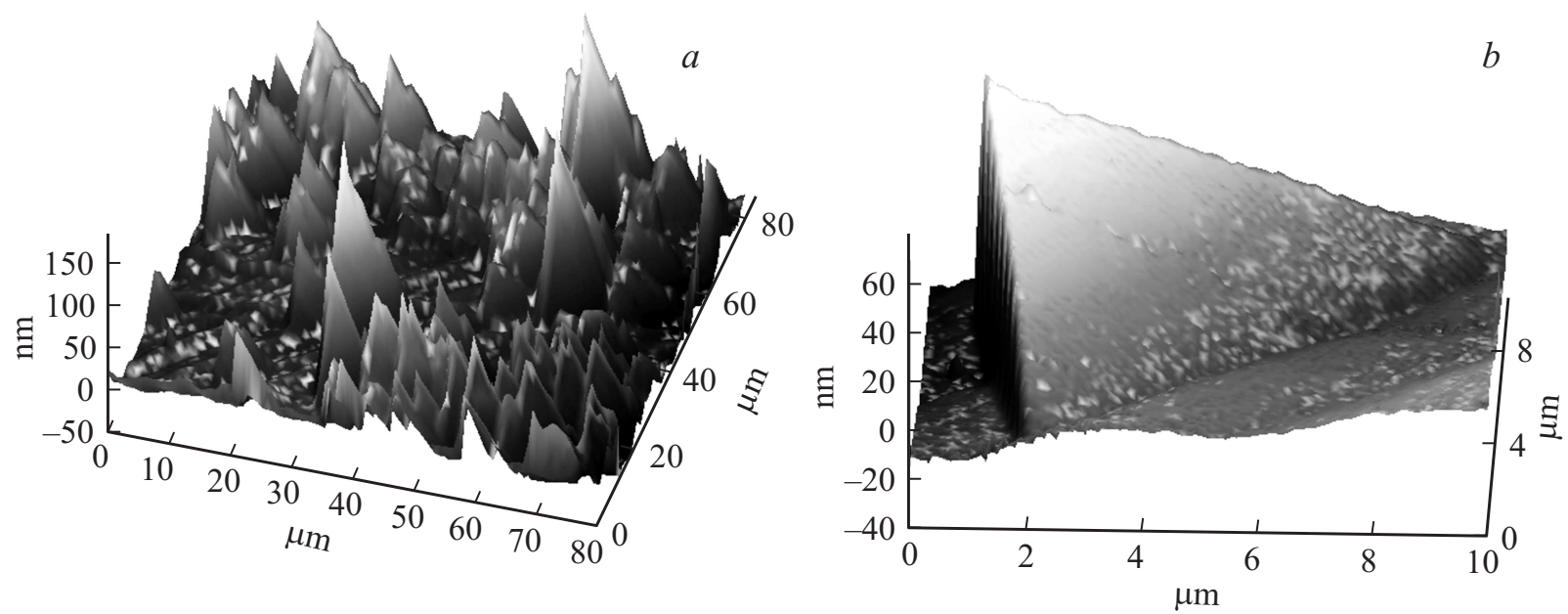

Рис. 3. Кадр поверхности подложки S02 после травления, полученный на атомно-силовом микроскопе NT-MDT Nanoeducator II; $b$ - вид отдельной террасы на поверхности подложки S02.

плоскости порядка 10 мкм и высотой до 100 нм. Исследование геометрической формы и профилей террас позволяет определить направление в плоскости и величину отклонения поверхности террас относительно поверхности подложки. Величины направления отклонения в плоскости образца $(\Delta \varphi)$ и в перпендикулярной плоскости $(\Delta \psi)$, определенные путем анализа данных АСМ и ИМБС, приведены в таблице вместе с дан-

Величины углов разориентации $(\Delta \varphi, \Delta \psi)$ алмазных подложек, определенные методами рентгеновской дифрактометрии, интерференционной микроскопии белого света и атомно-силовой микроскопии

\begin{tabular}{c|c|c|c|c|c|c}
\hline Образец & $\Delta \varphi$ ИМБС,$^{\circ}$ & $\Delta \varphi$ АСМ, ${ }^{\circ}$ & $\Delta \varphi$ РД, ${ }^{\circ}$ & $\Delta \psi$ ИМБС,${ }^{\circ}$ & $\Delta \psi{\text { АСМ },{ }^{\circ}} \Delta \psi$ РД, $^{\circ}$ \\
\hline S01 & 301 & 302 & 297 & 1.48 & 1.1 & 1.65 \\
S02 & 224 & 221 & 222 & 1.80 & 0.95 & 2.33 \\
S03 & 258 & - & 256 & 0.93 & - & 1.18 \\
S05 & 206 & - & 187 & 0.57 & - & 0.60
\end{tabular}


ными рентгеновской дифрактометрии. Обработка карт поверхности дает бо́льшую по сравнению с методом РД погрешность определения углов разориентации (для метода ИМБС - до $0.5^{\circ}$ по $\Delta \psi$ и до $20^{\circ}$ по $\Delta \varphi$ ). В пределах погрешности наблюдается совпадение углов разориентации, определенных методами РД и ИМБС. Для метода АСМ хорошее совпадение результатов наблюдается только для угла $\Delta \varphi$ поворота в плоскости, а величины $\Delta \psi$ заметно меньше, чем для РД или же ИМБС. Это отличие может быть объяснено двумя факторами. Во-первых, метод АСМ обладает лучшим разрешением и точнее прописывает вершины пиков террас, которые могут быть больше растравлены в плазме СВЧ разряда, что может уменьшать наклон плоскости террасы. Во-вторых, анализируемое методом АСМ поле для единичной террасы ограниченно $(10 \times 10$ мкм на рис. $3, b)$, что может давать систематическую погрешность при обработке кадра и определении угла наклона террасы относительно некоторой усредненной по размеру кадра поверхности подложки. В методе ИМБС поле кадра больше $(110 \times 110$ мкм $)$ и вычитание наклона усредненной поверхности подложки, по всей видимости, проводится корректнее. Из таблицы видно, что значения ИМБС также систематически немного меньше результатов, полученных методом РД, хоть и согласуются в пределах погрешности. Метод РД в данном случае выступает как эталонный, так как его точность определения углов разориентации заведомо выше, чем у ИМБС или АСМ. Сравнение результатов, проведенное в таблице, подтверждает сделанное предположение о том, что форма образующихся при травлении подложки терpac определяется кристаллографическими плоскостями. Поверхность террас с большой точностью совпадает с кристаллографической плоскостью (001). Дополнительные РД измерения показали, что, хотя верхняя грань террасы формируется плоскостью (001), боковые вертикальные грани сформированы плоскостями типа (110). Природа образования такой огранки, по всей видимости, объясняется анизотропией скорости травления монокристаллического алмаза в данных условиях. Значительное влияние на возможности формирования таких террас оказывает также и плотность дислокаций в подложке. Для подложек с плотностью дислокаций выше $10^{4} \mathrm{~cm}^{-2}$ при травлении в плазме $\mathrm{H}_{2}+\mathrm{O}_{2}$ наблюдается лишь образование множества ямок травления без формирования террас $[9,10]$. Эти обнаруженные в эксперименте эффекты остаются пока не объясненными. Отметим, что формируемые при травлении террасы можно использовать для экспресс-анализа разориентации и для повышения точности контроля угла отклонения при шлифовке подложек. Также при достижении низких значений угла разориентации применение процедуры анизотропного травления может позволить получать сингулярные (001) террасы макроскопических размеров, пригодные для дальнейшего роста на них гомоэпитаксиальных CVD пленок с заданными свойствами.

\section{4. Заключение}

В работе описаны способы экспериментальной диагностики и управления величиной отклонения плоскости поверхности монокристаллических алмазных НРНТ подложек относительно кристаллографических плоскостей (001). Такие подложки используются для гомоэпитаксиального выращивания как легированных, так и нелегированных пленок CVD алмаза. При травлении подложек в плазме СВЧ разряда в чистом водороде либо в водород-кислородной смеси наблюдалось формирование массивов сингулярных (001) террас. Сравнение результатов анализа геометрических параметров террас методами ИМБС и АСМ с результатами РД анализа ориентации кристаллографических осей позволило подтвердить предположение о том, что поверхности террас образованы кристаллографической плоскостью (001). Боковые грани террас образованы плоскостями типа (110). Это явление может быть использовано как для экспрессанализа ориентации подложки и повышения точности контроля угла отклонения при шлифовке подложек, так и для получения сингулярных (001) участков монокристаллических алмазных подложек макроскопических размеров.

Работа выполнена при финансовой поддержке гранта Российского научного фонда (проект № 16-19-00163). В работе использовано оборудование ЦКП ИФМ РАН „Физика и технология микро- и наноструктур“.

\section{Список литературы}

[1] R. Sussmann. CVD diamond for electronic devices and sensors (John Wiley\& Sons, 2009).

[2] E. Kohn, A. Denisenko. Thin Sol. Films, 515, 4333 (2007).

[3] A.B. Muchnikov, D.B. Radishev, A.L. Vikharev, A.M. Gorbachev, A.V. Mitenkin, M.N. Drozdov, Y.N. Drozdov, P.A. Yunin. J. Cryst. Growth, 442, 62 (2016).

[4] A.B. Muchnikov, A.L. Vikharev, J.E. Butler, V.V. Chernov, V.A. Isaev, S.A. Bogdanov, A.I. Okhapkin, P.A. Yunin, Y.N. Drozdov. Phys. Status Solidi A, 212, 2572 (2015).

[5] O.A. Ivanov, A.B. Muchnikov, V.V. Chernov, S.A. Bogdanov, A.L. Vikharev, J.E. Butler. Mater. Lett., 151, 115 (2015).

[6] A.L. Vikharev, A.M. Gorbachev, V.A. Koldanov, D.B. Radishchev. Plasma Phys. Rep., 31, 338 (2005).

[7] Д.М. Хейкер. Рентгеновская дифрактометрия монокристаллов (Л., Машиностроение, 1973).

[8] В.Н. Стасевич. Технология монокристаллов (М., Радио и связь, 1990).

[9] J. Achard, F. Silva, O. Brinza, X. Bonnin, V. Mille, R. Issaoui, M. Kasu, A. Gicquel. Phys. Status Solidi A, 206 (9), 1949 (2009).

[10] M. Naamoun, A. Tallaire, F. Silva, J. Achard, P. Doppelt, A. Gicquel. Phys. Status Solidi A, 209 (9), 1715 (2012).

Редактор А.Н. Смирнов 


\title{
Formation of singular (001) terraces on the surface of single-crystal HPHT diamond substrates
}

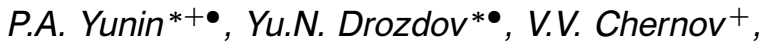 \\ V.A. Isaev ${ }^{+}$, S.A. Bogdanov ${ }^{+}$, A.B. Muchnikov ${ }^{+}$ \\ * Institute for Physics of Microstructures, \\ Russian Academy of Sciences, \\ 603950 Nizhny Novgorod, Russia \\ + Institute for Applied Physics, \\ Russian Academy of Sciences, \\ 603950 Nizhny Novgorod, Russia \\ - Lobachevsky State University of Nizhny Novgorod, \\ 603950 Nizhny Novgorod, Russia
}

\begin{abstract}
Techniques for diagnostics and control of misorientation angle for (001) vicinal single-crystal HPHT (High Pressure High Temperature) diamond substrates are discussed. The substrates are used for homoepitaxial growth of doped and undoped CVD diamond layers. Formation of singular (001) terraces on HPHT diamond surface after plasma etching in pure hydrogen or oxygen-hydrogen mixture is reported. This phenomenon can be used for express misorientation determination or improvement of miscut angle control precision during substrate polishing. It also opens up possibility for obtaining large singular (001) areas of single crystal diamond substrate.
\end{abstract}

